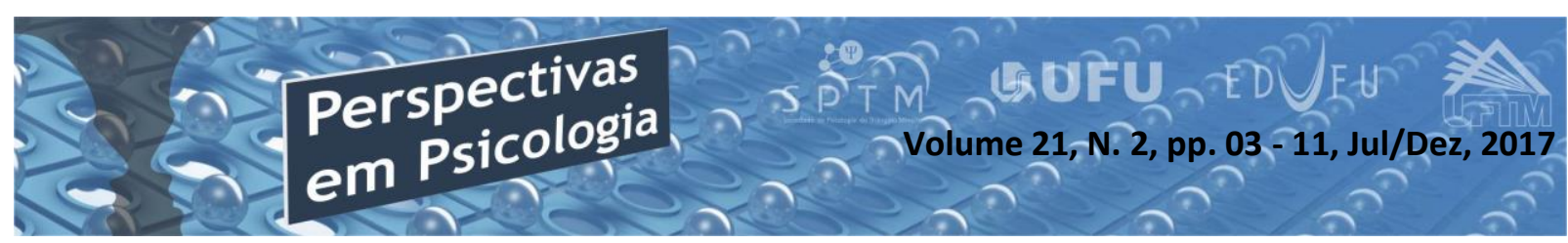

\title{
DISFUNÇÃO FAMILIAR ENTRE PESSOAS USUÁRIAS DE ÁLCOOL, TABACO E OUTRAS DROGAS
}

\author{
Rayrane Clarah Chaveiro \\ Roselma Lucchese \\ Ivania Vera \\ Bruno Souza Calixto \\ Heliana Batista de Oliveira \\ (Universidade Federal de Goiás, Regional Catalão - UFG/RC)
}

\begin{abstract}
Resumo
O uso abusivo de álcool, tabaco e outras drogas, tem se configurado um problema mundial de saúde, devido aos danos sociais, psíquicos e biológicos que podem provocar. O objetivo do estudo foi analisar a funcionalidade familiar das pessoas que abusam de substâncias psicoativas e identificar fatores relacionados. Estudo de corte transversal realizado em um município do sudeste goiano em clínicas de recuperação em dependência química e Centro de Atenção Psicossocial (CAPS). Variável desfecho foi Elevada Disfuncionalidade Familiar obtida segundo instrumento Adaptation, Partnership, Growth, Affection e Resolve, as variáveis independentes analisadas foram: sexo, escolaridade, estado civil, religião, ter se sentido triste ultimamente, se sentir uma pessoa inútil, sem préstimo. Participaram do estudo 266 indivíduos, dos quais $151(56,8 \%)$ apresentaram, de acordo com os scores boa funcionalidade familiar, e $20,3 \%$ e $22,9 \%$ apresentaram moderada disfuncionalidade familiar e elevada disfuncionalidade família. Não ter religião, sentimento de tristeza e inutilidade foram associados à elevada disfuncionalidade familiar. Sendo assim é de fundamental importância a participação da família na busca por mudanças do indivíduo que usa e abusa de álcool, tabaco e outras drogas.
\end{abstract}

Palavras- chave: alcoolismo; drogas ilícitas; relações familiares; tabaco; Enfermagem.

\section{Abstract \\ Family Dysfunction among People of Alcohol Users, Tobacco and other Drugs}

The abuse of alcohol, tobacco and other drugs, has set up a global health problem, due to the social, psychological and biological damage that can cause. The aim of the study was to analyze the familiar functionality of those who abuse psychoactive substances and identify related factors. cross-sectional study conducted in a municipality of goiano southeast recovering clinics in chemical dependency and Psychosocial Care Center (CAPS). Outcome variable was High Dysfunctionality Family obtained second instrument Adaptation, Partnership, Growth, Affection and Resolve, the independent variables were sex, education, marital status, religion, have lately felt sad, feel a useless person, useless. The study included 266 individuals, of which $151(56.8 \%)$ presented according to the scores good family functioning, and $20.3 \%$ and $22.9 \%$ had moderate and high dysfunctionality family dysfunctionality family. No religion, feelings of sadness and worthlessness were associated 
with high family dysfunction. Therefore it is of fundamental importance to family participation in the search for changes in individual use and abuse of alcohol, tobacco and other drugs.

Keywords: alcoholism; illicit drugs; family relationships; tobacco; nursing.

\section{Introdução}

$\mathrm{Na}$ sociedade do século XXI, é notória a grande mudança na relação do ser humano com as drogas, visto que inicialmente esta era utilizada em cerimônias específicas, como rituais religiosos. Com o advento da sociedade pós-moderna o consumo se modifica, e configura um modo desregrado e impulsivo, por indivíduos de diferentes idades, classe social, escolaridade, entre outros (Silva \& Silva, 2015).

O consumo abusivo de álcool, tabaco e outras drogas torna-se diariamente um grave problema de saúde e social, e tem sido uma questão amplamente divulgada e discutida. Os agravos provocados pelo uso dessas substâncias têm gerado inquietude mundial, tendo em vista os danos sociais, psíquicos e biológicos que estes podem provocar, sendo assim é relevante que a família exerça seu papel, vez que esta é considerada como principal suporte/auxílio a esses indivíduos que usam e abusam de álcool, tabaco e outras drogas (Who, 2014).
A família e o ambiente social que esta constitui, exerce forte influência no estilo de vida do jovem. O diálogo com os familiares possui essencial importância no que tange o comportamento do indivíduo, esteja esse relacionado ou não ao uso de drogas (Marconet e outros, 2008; Alencar \& Alencar, 2009).

O ambiente familiar é responsável por inserir seus membros na cultura, e instruí-los na construção de relações primárias, logo, esta influencia significativamente na reação do jovem perante aos perigos expostos pela sociedade. Atitudes e comportamentos, bem como os valores familiares são modelos importantes, visto que o ciclo familiar interfere na maneira como $o$ indivíduo constrói seus princípios ao longo de sua história (Bernardy e outros, 2011).

Diante da relevância da família no comportamento e modo de ser das pessoas, o presente estudo preocupou-se com a relação da família dos indivíduos que abusa de álcool e outras drogas, com a inquietação de como a funcionalidade familiar é analisada por estes? Neste sentido, objetivou-se analisar a funcionalidade familiar das pessoas em uso 
e abuso de substâncias psicoativas e identificar fatores relacionados.

\section{Métodos}

Delineamento, locais, população e período do estudo

Estudo de corte transversal conduzido com indivíduos que se encontravam em tratamento para dependência química em seis clínicas de reabilitação e um Centro de Atenção Psicossocial (CAPS) localizadas na região sudeste do Estado de Goiás, Brasil Central. A coleta dos dados ocorreu de agosto de 2013 a fevereiro de 2014.

Critérios de inclusão e exclusão

Pessoas, maiores de 18 anos, em processo de reabilitação em clinicas de recuperação ao usuário de substâncias químicas vinculadas ao projeto maior, além de integrantes de grupos Álcool e Drogas (AD), atendidos pelo Centro de Atenção Psicossocial (CAPS). Foram excluídos da coleta os indivíduos que se encontravam sedados ou que apresentavam sintomas de confusão mental no momento da entrevista.

Coleta de dados
Durante o período do estudo, todos os indivíduos em tratamento nas instituições e, que atendiam os critérios de legibilidade, foram convidados a participar do mesmo configurando uma amostra por conveniência. Após assinatura do Termo de Consentimento Livre e Esclarecido (TCLE), os participantes foram entrevistados, em local privativo.

\section{Variáveis de desfecho}

A variável desfecho foi a Elevada Disfuncionalidade Familiar obtida segundo o escore do instrumento Adaptation, Partnership, Growth, Affection e Resolve (APGAR) (Smilkstein, 1978). O instrumento APGAR de família é um questionário realizado por Gabriel Smilkstein usado na identificação da função familiar durante as consultas médicas (Smilkstein, 1978). Possibilita a avaliação da satisfação subjetiva com o cuidado recebido do membro familiar por meio de cincoaspectos dopapel da família: Adaptation (Adaptação), Partnership (Companheirismo), Growth (Desenvolvimento), Affection (Afetividade) e Resolve (Capacidade resolutiva) (Smilkstein, 1978; Duarte, 2001; Brasil, 2007).

Os aspectos são respondidos com as seguintes escolhas com pontuações distintas.Para cada um dos cinco itens, são 
atribuidos escores sendo elas: sempre, as vezes e nunca cujas pontuações são 2,1 e 0 respectivamente. Somando se o total dos 5 itens o total dos escores vão de 0 a 10 pontos, considerando: Elevada Disfuncionalidade Familiar (EDF) - 0 a 4; Moderada Disfuncionalidade Familiar (MDF) - 5 e 6 e, Boa Funcionalidade Familiar (BFF) $\quad-\quad 7$ a 10 pontos (Smilkstein, 1978; Duarte, 2001; Brasil, 2007).

\section{Variáveis de predição}

As variáveis independentes analisadas foram: sexo, categorizada pelo gênero; escolaridade, categorizada por $>7$ ou $\leq 7$; estado civil, categorizada por vive com o companheiro e vive sem o companheiro; religião, categorizada por ter ou não ter religião;ter se sentido triste ultimamente, questionou-se se nos últimos 30 dias se sente triste a maioria do tempo; se sentir uma pessoa inútil, sem préstimo questionou-se se nos últimos 30 dias se sente sem utilidade para os outros ou para si mesmo, na maior parte do tempo.

\section{Análise estatística}

Os dados do questionário foram transpostos e analisados no programa
Statistical Package for the Social Sciences (SPSS), versão 22). $\mathrm{Na}$ análise descritiva realizou-se a estimativa em percentagem e intervalo de confiança de 95\% (IC:95\%). Em seguida precedeu-se a regressão logística simples com Odds Ratio (OR) e IC95\%. O teste de qui-quadrado $\left(\chi^{2}\right)$ foi utilizado para verificar as diferenças entre as proporções e, valores de $\mathrm{p}<0,05$ foram considerados estatisticamente significantes.

\section{Aspectos éticos}

Este estudo foi aprovado no Comitê de Ética em Pesquisa da Universidade Federal de Goiás, protocolo nํ162/2012 e respeitou os princípios éticos da Resolução n-466/2012 que regula os princípios éticos com pesquisas em seres humanos no Brasil. Todos os participantes assinaram o TCLE.

\section{Resultados}

Participaram do estudo 266 indivíduos, dos quais $151 \quad(56,8 \%)$ apresentaram de acordo com os scores do APGAR BFF, e $20,3 \%$ e $22,9 \%$ apresentaram MDF e EDF respectivamente. Como o exposto na tabela 1. 
RAYRANE CLARAH CHAVEIRO, ROSELMA LUCCHESE, IVANIA VERA, BRUNO SOUZA CALIXTO, HELIANA BATISTA DE OLIVEIRA

Tabela 1 Descrição da Funcionalidade Familiar entre indivíduos que abusam de álcool e outras drogas. Brasil Central, 2014

\begin{tabular}{ccc}
\hline VARIÁVEL & $\mathbf{n}(\boldsymbol{\%})$ & IC (95\%) \\
\hline BFF & & \\
Sim & $151(56,8)$ & $(51,1-62,4)$ \\
Não & $115(43,2)$ & $(37,6-48,9)$ \\
MDF & & \\
Não & $212(79,7)$ & $(74,8-84,6)$ \\
Sim & $54(20,3)$ & $(15,4-25,2)$ \\
EDF & & $(71,8-82,0)$ \\
Não & $205(77,1)$ & \\
Sim & $61(22,9)$ & $(18,0-28,2)$ \\
\hline
\end{tabular}

Contudo, entre as categorias da família disfuncional, a de maior prevalência foi a EDF. Diante deste achado optou-se por esta variável como desfecho e analisar os fatores associados, como descritos na tabela 2 .

Tabela 2 Prevalência de elevada disfuncionalidade familiar e fatores associados em indivíduos que abusam de álcool e outras drogas. Brasil Central, 2014

\begin{tabular}{|c|c|c|c|}
\hline Variável & EDF & $\mathrm{OR}^{\dagger}(\mathbf{I C} 95 \%)^{\ddagger}$ & $\mathbf{p}$ \\
\hline \multicolumn{4}{|l|}{ Sexo } \\
\hline Masculino & $52(23,0)$ & 1,0 & \\
\hline Feminino & $8(20,5)$ & $0,8(0,3-1,9)$ & 0,7 \\
\hline \multicolumn{4}{|l|}{ Escolaridade } \\
\hline$>7$ anos & $35(20,8)$ & 1,0 & \\
\hline$\leq 7$ anos & $26(26,5)$ & $1,3(0,7-2,4)$ & 0,2 \\
\hline \multicolumn{4}{|l|}{ Estado Civil } \\
\hline Vive sem companheiro & $50(23,3)$ & 1,0 & \\
\hline Vive com companheiro & $11(21,6)$ & $0,9(0,4-1,8)$ & 0,7 \\
\hline \multicolumn{4}{|l|}{ Religião } \\
\hline Tem & $45(20,1)$ & 1,0 & \\
\hline Não tem & $15(36,6)$ & $2,2(1,1-4,6)$ & $\mathbf{0 , 0 2}$ \\
\hline \multicolumn{4}{|c|}{ Tem se sentido triste ultimamente } \\
\hline Não & $17(15,6)$ & 1,0 & \\
\hline Sim & $44(28,0)$ & $2,1(1,1-3,9)$ & $\mathbf{0 , 0 1}$ \\
\hline \multicolumn{4}{|c|}{ Se sente uma pessoa inútil, sem } \\
\hline \multicolumn{4}{|l|}{ préstimo } \\
\hline Não & $46(19,7)$ & 1,0 & \\
\hline Sim & $15(45,5)$ & $3,3(1,5-7,2)$ & $<0,01$ \\
\hline
\end{tabular}


Após a análise univariada, houve associação com o desfecho nas seguintes variáveis preditoras: religião $\mathrm{p}=0,02(\mathrm{OR}$ 2,2 [IC 95\%: 1,1-4,6]); tem se sentido

\section{Discussão}

Em um estudo realizado com usuários que abusavam ou eram dependentes químicos em centros de tratamento no Chile, houve prevalência da BFF (66,4\%), como o encontrado neste estudo, seguida de MDF $(22,4 \%)$ e EDF (11,2\%) (Musso e outros, 2012).

No presente estudo a variável não ter religião foi associada a EDF. Este se relaciona com outro estudo que afirma que indivíduos que possuem religião e/ou familiares praticantes de alguma religião estão menos propensos a envolvimento com álcool e drogas, visto que possuem uma melhor relação intrafamiliar, em contrapartida o mesmo estudo aponta que indivíduos que relataram religião espírita em comparação a outras religiões, apresentam maior consumo de álcool, configurando-se em um grupo de risco (Funai \& Pillon, 2011).

Em uma pesquisa realizada com 241 estudantes de enfermagem, 24\% dos entrevistados relataram o uso da atual de substâncias psicoativas, $15 \%$ relataram abuso/dependência provável. Logo, aqueles que eram mais religiosos tenderam triste ultimamente $\mathrm{p}=0,01 \quad(\mathrm{OR}: 2,1 \quad[\mathrm{IC}$ 95\%: 1,1-3,9]); se sente uma pessoa inútil, sem préstimo $\mathrm{p} \leq 0,01$ (OR: 3,3 [IC 95\%: 1,5-7,2]).

a ter menores taxas de prevalência de consumo de substâncias. Nesta direção se considera um fator de proteção para uso de drogas, ter uma religião. Sendo assim, incentivar jovens e adolescentes a frequentar instituições como igrejas e/ou associações religiosas é um fator de proteção/prevenção para uso de álcool, tabaco e outras drogas visto que são locais de intensa interação social, e estes influenciam na construção da personalidade dos indivíduos e incentivam a busca da vida digna (Gnadt, 2006; Pillon e outros, 2011).

No que tange ao sentimento de tristeza relacionada a relações familiares, neste estudo a variável se sentir triste e se sentir inútil foram associadas a variável EDF, corroborando com este achado temse um estudo que estimou $10 \%$ de sua amostra, demonstravam tristeza e sintomatologia depressiva. Igualmente, relataram relacionamento familiar como ruim, fraco apoio emocional e baixa interação positiva (Avanci e outros, 2008).

Um estudo realizado em 2014 objetivou analisar as taxas do consumo de tabaco e ingestão de álcool em termos de escala de funcionamento familiar (APGAR), os dados mostraram relação 
estatisticamente significativa em todos os casos ( $\leq .0,05)$. Em $10 \%$ da amostra, adolescentes que apresentaram EDF, tiveram valores baixos e médio de dependência nicotínica. Contudo, $50 \%$ dos indivíduos que relataram de MDF, apresentaram alta dependência ao tabaco e seus derivados. Ainda neste estudo afirmase que a ingestão frequente de ambos os elementos afeta negativamente o ambiente familiar (Zurita \& Álvaro, 2014).

\section{Conclusão}

A presente pesquisa quando comparada a outros estudos, confirma o fato de que entre a população usuária de drogas prevalece maior índice de funcionalidade familiar, entretanto difere quando apresenta EDF mais prevalente que a MDF, como o relatado em diferentes pesquisas. $\mathrm{O}$ estudo mostrou que não ter religião e sentimento de tristeza e inutilidade foram associados a EDF. O que se configura, que os indivíduos que declararam não ter religião tiveram mais chances de não ter uma boa relação familiar. Assim como aqueles que declararam se sentir triste ou se sentir inútil.

Configurando-se assim, o quão é fundamental a participação e apoio da família à usuários que usam e abusam de álcool, tabaco e outras drogas na busca por tratamento, vez que estes se encontram sozinhos, desamparados e sem perspectiva para mudanças, logo o apoio e carinho familiar se faz necessário na busca por transformações positivas.

Por fim reconhece-se as limitações desta pesquisa por se tratar de um estudo transversal e inviabilizar inferências ás causas e efeitos da EDF. Também a amostra por conveniência não favores a generalização dos dados. Contudo apresenta-se como um estudo que estimou a prevalência da EDF como ponto a ser considerado em futuras pesquisas e na atenção à saúde destas famílias.

\section{Referências}

Alencar, D.C. \& Alencar, A.M.P.G. (2009). O papel da família na adaptação do adolescente diabético. Rev Rene. 10(1), 19-28.

Avanci, J. Q., Assis, S. G. \& Oliveira, R.V. C. (2008). Sintomas depressivos na adolescência: estudo sobre fatores psicossociais em amostra de escolares de um município do Rio de 
Janeiro, Brasil. Cad. Saúde Pública. 24(10), 2334-2346. https://doi.org/10.1590/S0102$311 X 2008001000014$.

Brasil (2007). Ministério da Saúde. Secretária de atenção à saúde. Departamento de atenção básica. Envelhecimento e saúde da pessoa idosa. Brasília.

Bernardy, C. C. F., Oliveira, M. L. F. \& Belini, L. M. (2011). Jovens infratores e a convivência com drogas no ambiente familiar. Rev Rene.12(3), 589-96.

Duarte, Y. A. O. (2001). Família: rede de suporte ou fator estressor. A ótica de idosos e cuidadores familiares. São Paulo. 196f. Tese (Doutorado) - Universidade de São Paulo Escola de Enfermagem.

Ferreira, S. C. \& Machado, R. M. (2013). Equipe de saúde da família e o uso de drogas entre adolescentes. Cogitare Enferm. 18(3), 482-489. https://doi.org/10.5380/ce.v18i3.33560.

Gnadt, B. (2006). Religiousness, current substance use, and early risk indicators for substance $\begin{array}{lllll}\text { abuse in } \quad \text { nursing } & \text { students. } J \text { AddictNurs. 17(3), }\end{array}$ https://doi.org/10.1080/10884600600862103.

Marcon, S.S., Navarro, F.M., Hayakawa, L.Y., Scardoelli, M.G.C. \& Waidman, M.A.P. (2008). Relações familiares ante os valores e costumes em diferentes etnias. Rev Rene. 9(2), 9-19. https://doi.org/10.1157/13098670.

Milena, A. P.; Milena, R. P.; Fernández, M. L. M.; Helmling, F. J. L.; Gallardo, I.M.; Pulido, I. J. (2007). Estructura y funcionalidad de la familia durante la adolescencia: relación con el apoyo social, el consumo de tóxicos y el malestar psíquico. Aten Primaria. 39(2), 61-7. https://doi.org/10.1157/13098670

Musso, L. B. e outros. (2012). El distrés psicológico y abuso de drogas en pacientes en centros de tratamiento del granvalparaíso - chile: implicaciones para las políticas y los programas. Texto Contexto Enferm. 21, 114-123. https://doi.org/10.1590/S010407072012000500015 .

Pillon, S.C.; Santos, M.A.; Gonçalves, M.A.S.; Araújo, K.M. (2011). Uso de álcool e espiritualidade entre estudantes de enfermagem. Rev Esc Enferm USP. 45(1), 100-107. https://doi.org/10.1590/S0080-62342011000100014.

Silva, R, K. A. \& Silva, S. V. A. (2015). "classe- que- vive- do-trabalho" e o uso de álcool e outras drogas: uma necessária problematização no contexto da reestruturação produtiva do capital. Caderno Discente ESUDA, 2(1).

Smilkstein, G. (1978). The family APGAR: a proposal for family function test and its use by physicians. J FamPrac. 6(6),1231-1239. 
Who (2014). World Health Organization. Global status report on alcohol and health 2014.Geneva,

Switzerland.Fromhttp://apps.who.int/iris/bitstream/10665/112736/1/9789240692763_eng .pdf?ua $=1$

Zurira, F. \& Álvaro, J. I. (2014). Repercusión del tabaco y alcohol sobre factores académicos y familiares en adolescentes. Health and Addictions. 14(1), 59-70.

\section{Os autores:}

Rayrane Clarah Chaveiro aluna do curso Enfermagem da Universidade Federal de Goiás - Regional Catalão. Atuou como aluna PIBIC durante o ano de 2014. E-mail: rayraneclarah16@hotmail.com

Roselma Lucchese é Enfermeira. Doutora em Enfermagem/EEUSP. Docente no programa de Pós-Graduação em Gestão Organizacional da Universidade Federal de Goiás. Prof. titular na UFG - Regional Catalão. E.mail: roselmalucchese@ hotmail.com

Ivania Vera é enfermeira, doutora em Enfermagem com ênfase à saúde do idoso (FEN/UFG). Universidade Federal de Goiás - Regional Catalão (UFG/CAC). Departamento de Enfermagem da Universidade Federal de Goiás - Regional Catalão (UFG/CAC). Pesquisadora e vice-líder do Grupo de Pesquisa Gestão, Ensino e Cuidado em Saúde e Enfermagem (GENCSE). Orientadora no Programa de Pós-Graduação - Mestrado Profissional Gestão Organizacional (UFG/CAC). Av. Lamartine Pinto de Avelar, 1120, Setor Universitário - CEP 75704-020. Catalão, GO, Brasil (64) 96451910/3441-5300. E.mail: ivaniavera@ gmail.com

Bruno Souza Calixto é enfermeiro graduado pela Universidade Federal de Goiás, Regional Catalão. Email:brunodesouzacalixto@yahoo.com.br

Heliana Batista de Oliveira possui graduação em Ciências Biológicas, mestrado e doutorado em Imunologia e Parasitologia Aplicadas. Atualmente é professora Associado nível 1 da Universidade Federal de Goiás Regional Catalão. E.mail: helianalia@gmail.com

Recebido em: 03/09/2016.

Aprovado em: 20/01/2017. 